\title{
REVIEW
}

\section{Recipes for adult stem cell plasticity: fusion cuisine or readymade?}

\author{
M R Alison, R Poulsom, W R Otto, P Vig, M Brittan, N C Direkze, M Lovell, T C Fang, S L Preston, \\ N A Wright
}

J Clin Pathol 2004;57:113-120

A large body of evidence supports the idea that certain adult stem cells, particularly those of bone marrow origin, can engraft at alternative locations, particularly when the recipient organ is damaged. Under strong and positive selection pressure these cells will clonally expand/ differentiate, making an important contribution to tissue replacement. Similarly, bone marrow derived cells can be amplified in vitro and differentiated into many types of tissue. Despite seemingly irrefutable evidence for stem cell plasticity, a veritable chorus of detractors has emerged, some doubting its very existence, motivated perhaps by more than a little self interest. The issues that have led to this situation include the inability to reproduce certain quite startling observations, and extrapolation from the behaviour of embryonic stem cells to suggest that adult bone marrow cells simply fuse with other cells and adopt their phenotype. Although these issues need resolving and, accepting that cell fusion does appear to allow reprogramming of haemopoietic cells in special circumstances, criticising this whole new field because some areas remain unclear is not good science.

See end of article for authors' affiliations

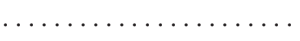

Correspondence to: Professor M Alison, Department of Histopathology, Hammersmith Hospital, Du Cane Road, London W12 ONN, UK; m.alison@ imperial.ac.uk

Accepted for publication 11 June 2003
T he study of embryonic stem (ES) cells for over 20 years has established the amazing flexibility of these cells, which are essentially able to generate almost all cells that arise from the three germ layers. However, since the publication of two papers in 1998, describing the growth in vitro of human ES derived either from the inner cell mass of the early blastocyst, ${ }^{1}$ or the primitive gonadal regions of early aborted fetuses, ${ }^{2}$ the field of stem cell research has gone into overdrive. There is now a genuine belief that stem cell research will deliver a revolution in terms of how we treat cardiovascular disease, neurodegenerative disease, cancer, diabetes, and the like. Regenerative medicine has been grabbing many of the headlines in both the biomedical and popular press over this period; not just concerning ethical issues, but also regarding the therapeutic potential of ES versus adult stem cells, although we suspect that sometimes a view on this last issue may depend upon one's moral standpoint.

\footnotetext{
"There is now a genuine belief that stem cell research will deliver a revolution in terms of
}

how we treat cardiovascular disease, neurodegenerative disease, cancer, diabetes, and the like"

Within the past year, doubt has been cast upon claims that certain adult stem cells, particularly those from the bone marrow, can jump lineage boundaries to generate completely new types of cells. This has led to a flurry of headlines along the lines of "Cell fusion leads to confusion", ${ }^{3}$ "Biologists question adult stem cell versatility", ${ }^{4}$ "Plasticity: time for a reappraisal?",, "Is transdifferentiation in trouble?", ${ }^{6}$ and "Are somatic stem cells pluripotent or lineage restricted?" Here, we will examine the contentious issues.

TO FUSE OR NOT TO FUSE: NO AND YES Claims for so called adult stem cell plasticity often rely on the appearance of Y chromosome positive cells in a female recipient of a bone marrow transplant from a male donor (fig 1). Alternatively, markers such as LacZ or green fluorescent protein (GFP) have been used, and these techniques are usually combined with lineage markers in attempts to demonstrate that there has been a switch in the fate (transdifferentiation) of the transplanted cells. A rapidly growing number of papers suggest that adult bone marrow cells can differentiate into many types of tissue, including skeletal muscle, cardiomyocytes and endothelia, neurones and glia, hepatocytes and bile duct epithelia, renal epithelia and podocytes, and gut mucosal cells and associated myofibroblasts (reviewed by Poulsom et al). ${ }^{8}$

However, two publications in Nature in 2002 alerted investigators to the possibility that perhaps all instances of transdifferentiation were the result of the fusion of bone marrow cells with the differentiated cells in the new organ, for example, the liver. One describes the consequences of mixing genetically altered bone marrow from GFP transgenic mice with ES cells; a very small proportion of the bone marrow cells fused with ES cells (2-11 hybrid clones $/ 10^{6}$ marrow cells) and these cells could subsequently adopt many of the phenotypes typical of ES cell

Abbreviations: CNS, central nervous system; CRE, CAMP response element; DAPI, 4,6-diaminidino-2 phenylindole; EGFP, enhanced green fluorescent protein; EPC, endothelial progenitor cell; ES, embryonic stem cell; GFP green fluorescent protein; NTBC, 2-(2-nitro-4-trifluoromethylbenzoyl)-1,3 cyclohexanedione; SMC, smooth muscle cell 


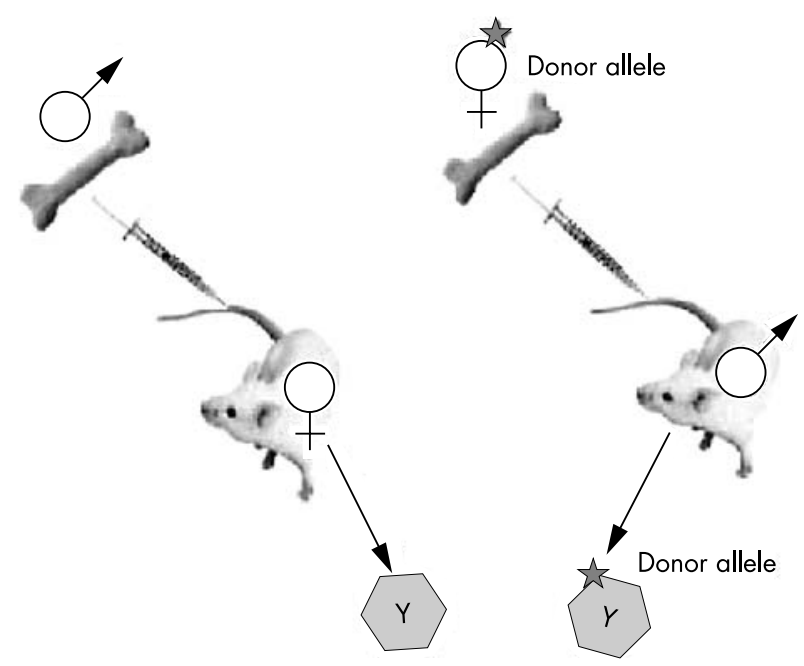

Figure 1 Tracking bone marrow by sex mismatched bone marrow transplantation. Male to female transplantation is useful for detecting transdifferentiation by detection of the $Y$ chromosome. However, cell fusion may be missed because not all chromosomes might be visible in the tissue section. Female to male transplantation is more suitable for detecting fusion events; marked bone marrow derived parenchymal cells with a $Y$ chromosome have definitely been formed by fusion with host cells.

differentiation. ${ }^{9}$ A similarly low frequency of fusion (one event $/ 10^{5}$ central nervous system (CNS) cells) was seen when mouse CNS cells (also genetically altered) were mixed with ES cells, and here the derived hybrid cells were able to show multilineage potential when injected into blastocysts, most prominently into liver. ${ }^{10}$

So what are the implications of cell fusion for stem cell biology? Various commentators at the time leapt to the assumption that all examples of apparent transdifferentiation were the result of cell fusion- "scientists are generating freak cells" opined the science editor of a UK broadsheet The Daily Telegraph (14/03/02). This, as we shall see, is patently not the case, although there are instances where cell fusion has been responsible for reprogramming of the haemopoietic stem cell gene expression pattern-not necessarily a bad thing if it has "cured" a potentially fatal metabolic disease! However, the two publications reporting cell fusion made it almost mandatory that investigators look at the karyotype of cells claimed to have been generated from the tissue of another type. Yet, if bone marrow cells naturally fused with other cell types, we might all have substantial complements of polyploid cells in many organs; this is patently not the case, except for perhaps in the liver and pancreas. In the liver, polyploidy, which develops early in life, is not generated by cell fusion. For example, in the rat the 4th postnatal week of life signals a change in cell composition, with the emergence of binucleate hepatocytes with two diploid nuclei, at a time when mononuclear diploid hepatocytes are disappearing: a widespread failure of cytokinesis appears to be responsible (fig 2). During further developmental growth, the component nuclei in these binucleate hepatocytes undergo simultaneous DNA replication, but then the two sets of chromosomes deploy upon a common spindle and cytokinesis yields two mononuclear daughter cells with $4 \mathrm{n}$ nuclei. Thus, the ploidy class of the nuclei of the preceding binucleate hepatocytes is always one class below the ploidy of the succeeding mononuclear cells, and the process is associated with a decrease in binucleate cell number. So cell fusion is not the cause of the postnatal development of polyploidy.

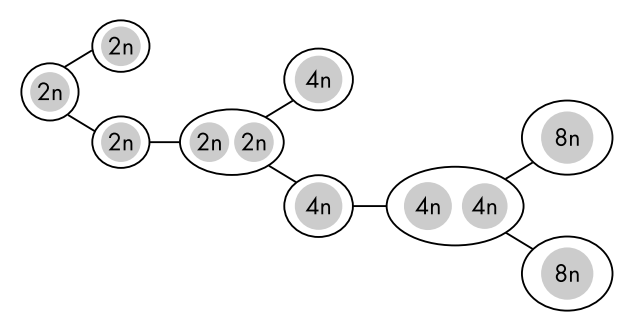

Figure 2 The early postnatal development of hepatocyte polyploidy is through the failure of cytokinesis, leading to the formation of binucleate cells (see text). This is quite distinct from the fusion of bone marrow cells with hepatocytes described in the Fah-/- mouse. ${ }^{11} 12$

Because most observations have been made in cases of sex mismatched bone marrow transplantation (donor male bone marrow to female recipient; fig 1 ), the obvious step is to examine the cells for the presence of $\mathrm{X}$ and $\mathrm{Y}$ chromosomes: if fusion was responsible then the donor (apparently) transdifferentiated cell would have an XXXY karyotype rather than $\mathrm{XY}$ of a purely transdifferentiated male donor haemopoietic stem cell. The major drawback to this type of analysis is that most are performed on paraffin wax embedded tissue sections of finite thickness-even in routine 4-6 $\mu \mathrm{m}$ thick sections of male control tissue, the Y chromosome (usually located at the nuclear periphery) is only detected in $50-60 \%$ of male cells, thus, the likelihood of "missing" the extra chromosomes present in a fusion cell is very real. This was not a problem encountered by Tran et al, ${ }^{13}$ who analysed almost 10000 thin buccal cells from five female recipients of $\mathrm{CD} 34+$ bone marrow cell transplants from male donors. Most importantly, they could detect Y chromosomes in $98 \%$ of male control cells and found two X chromosomes in $99 \%$ of female control cells. The number of $\mathrm{Y}$ and cytokeratin 13 doubly positive buccal cells in the female recipients ranged from $0.8 \%$ to $12.7 \%$, with only one XXXY cell $(0.01 \%)$ and one XXY cell $(0.01 \%)$ detected, both of which could have arisen by fusion. Not unreasonably, the authors concluded that bone marrow derived cells could transdifferentiate directly into buccal cells in the absence of cell fusion.

\section{"The two publications reporting cell fusion made it almost mandatory that investigators look at the karyotype of cells claimed to have been generated from the tissue of another type"}

There are numerous studies claiming, with some justification, that in many circumstances, cell fusion is not a major player in the reprogramming of bone marrow derived cells, although we should add the caveat that in studies relying on chromosome counts, extra chromosomes could have been missed during the tissue sectioning, thus underscoring the contribution made through cell fusion. Following on from animal studies illustrating massive replacement of infarcted myocardium by bone marrow derived cells, ${ }^{14}$ several impressive claims have been made for the therapeutic potential of autologous bone marrow for the treatment of myocardial infarction and heart failure in humans, ${ }^{15-17}$ and much effort has been expended in determining the mechanism of apparent transdifferentiation within the cardiovascular system. Caplice and colleagues ${ }^{18}$ examined the contribution of bone marrow derived cells to atherosclerotic plaques in human coronary arteries in eight cases of sex mismatched transplantation, finding that up to $10 \%$ of intimal smooth muscle cells (SMCs) were so derived. This figure was 100 fold greater than in non-diseased segments of the same vessels, 
and using probes for $\mathrm{X}, \mathrm{Y}$, and chromosome 18 failed to find evidence of polyploidy among thousands of SMCs. Using the same probes, 180 apparently transdifferentiated cardiomyocytes were found among 80000 cardiomyocytes $(0.23 \%)$ analysed from four female recipients of male bone marrow, again with no evidence that fusion had been responsible. ${ }^{19}$ Also relating to the cardiovascular system, endothelial progenitor cells (EPCs) can be derived ex vivo from human peripheral blood mononuclear cells, and these EPCs will transdifferentiate into functional cardiomyocytes when cocultured and in contact with rat cardiomyocytes; fusion was discounted because this could also occur when EPCs were in contact with fixed (dead) cardiomyocytes. ${ }^{20}$

In terms of stem cell plasticity, no field is more muddied than the CNS. The ability of brain cells to make blood cells and vice versa has been questioned (vide infra), and the cell fusion issue is far from resolved. For example, in wild-type mice transplanted with bone marrow from GFP transgenic animals, cells expressing both GFP and either neuronal or astrocytic markers have been found in the spinal cord and dorsal root ganglia; because these doubly positive cells had the same DAPI (4,6-diaminidino-2 phenylindole) fluorescence intensity (DNA content) as endogenous neurones and astrocytes, cell fusion was discounted as the operative mechanism. ${ }^{21}$ In contrast, in human female recipients of male bone marrow transplants, although only four Purkinje cells with a Y chromosome were found among 5860 Purkinje cells $(0.068 \%)$ observed in four recipients, one of these $\mathrm{Y}$ positive cells had two X chromosomes (the others had one $\mathrm{X}$ and one $\mathrm{Y}$ chromosome). ${ }^{22}$ Because the full complement of two $\mathrm{X}$ chromosomes could only be detected in one in five Purkinje cells in control female cerebellum, the authors thought that they may have missed the sex chromosomes in the transdifferentiated cells, and thus seemed to favour cell fusion as an explanation for their limited observationshardly conclusive evidence either way! Yet, given the structural complexity of Purkinje cells, transdifferentiation and integration de novo would be astonishing.

Diabetes is a major cause of morbidity and mortality in the Western world, and islet cell transplantation technology is still in its infancy. Thus, the possibility that bone marrow may fit the bill as a pancreatic stem cell is very attractive; surprisingly, evidence for this has, for one reason or another, only recently been forthcoming. Adopting a very elegant genetic approach, male mouse bone marrow cells transplanted into lethally irradiated female recipients were found to transdifferentiate, without fusion, into pancreatic islet cells possessing many markers of $\beta$ cell differentiation and the ability to secrete insulin in response to glucose..$^{23}$ In the first set of experiments, donor mice were generated by crossing mice that had cAMP response element (CRE) recombinase under the control of the insulin 2 promoter (INS2-CRE) with mice that expressed enhanced GFP (EGFP) at the ROSA26 locus preceded by 3 floxed translation stop codons (ROSA-stoplox-EGFP). Pancreatic $\beta$ cells in these animals expressed EGFP because of activation of the insulin promoter and the subsequent expression of CRE, which removes the chromosomal portion flanked by the LoxP sites: transplanted male bone marrow cells from these mice were found in the pancreatic islets of wild-type female recipients possessing the $\mathrm{Y}$ chromosome and expressing EGFP. Of course, cell fusion could not be ruled out, so in a second approach bone marrow from INS2-CRE male mice was transplanted into irradiated ROSA-stoplox-EGFP female recipients: chromosome $\mathrm{Y}$ and insulin doubly positive cells were found in the recipient pancreatic islets, but there was no EGFP expression, indicating that no donor INS2-CRE cells had fused with ROSA26-stoplox-EGFP recipient cells.
In the kidney too, transdifferentiation, this time from a clonal population derived from a highly purified bone marrow stem cell into functional mesangial cells, could not be ascribed to cell fusion. ${ }^{24}$ Performing male to male transplants (GFP positive to wild-type) resulted in numerous GFP positive mesangial cells, and careful inspection failed to find more than one Y chromosome in any of them.

Although not directly seeking to establish the presence or absence of fusion as an explanation for transdifferentiation, several other observations are consistent with fusion being an unlikely mechanism. An investigation by fluorescence in situ hybridisation of the karyotype of male donor peripheral blood stem cells that had apparently transdifferentiated into epidermal, hepatic, and gastric mucosal cells in human female recipients clearly found only one $\mathrm{X}$ and one $\mathrm{Y}$ chromosome in each of these cells. ${ }^{25}$ Similarly, Okamoto et al found no evidence for cell fusion being responsible for the apparent engraftment and differentiation of bone marrow cells into mucosal epithelial cells throughout the gastrointestinal tract of human female recipients of male bone marrow. ${ }^{26}$ They noted sustained engraftment over many months/years, with up to $13 \%$ (much higher than the reported fusion rates with ES cells) of colonocytes being marrow derived shortly after the development of graft versus host disease, but more importantly Y chromosome positive epithelia did not stain more intensely than other epithelial cells with DAPI.

We can also look at epithelial tissue from mothers of male offspring where postpartum exacerbation of thyroiditis could be the result of transplacentally acquired fetal cells that cause an alloimmune disease, previously regarded as an autoimmune disease. ${ }^{27}$ Particularly noteworthy was one female patient with clusters of fully differentiated thyroid follicular cells bearing one $\mathrm{X}$ and one $\mathrm{Y}$ chromosome; of course, the source of the transdifferentiated cells was the fetus rather than a deliberate transplant, but nevertheless, no follicular cells were XXXY, suggesting that cell fusion was not responsible for the phenomenon, even when fetal cells were the source.

Perhaps a better approach to the study of the ploidy of transdifferentiated cells is indicated by the studies of Kleeberger et al, ${ }^{28}{ }^{29}$ who examined liver and lung chimaerism in human allografts by polymerase chain reaction analysis of a highly polymorphic tetranucleotide repeat marker at the human $\beta$ actin related pseudogene. Using laser assisted microdissection of small areas of pure parenchymal cells they did indeed find that almost all samples displayed the genotype of both donor and recipient, thus suggesting cell engraftment from circulating multipotent bone marrow precursors. However, cell fusion could not be excluded because the samples contained more than one cell, but if single cells could be captured, this approach could readily resolve questions regarding cell fusion.

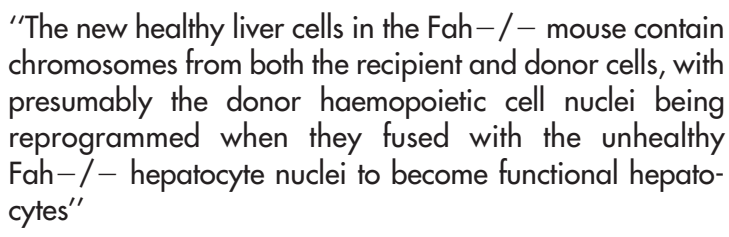

Now for the twist in the tail! In undoubtedly the most convincing "proof of principle" demonstration of the potential therapeutic usefulness of bone marrow, mice with a metabolic liver disease have been cured. ${ }^{30}$ Female mice deficient in the enzyme fumarylacetoacetate hydrolase (Fah-/-, a model of fatal hereditary tyrosinaemia type 1), a key component of the tyrosine catabolic pathway, can be 
rescued biochemically by $1 \times 10^{6}$ unfractionated bone marrow cells that are wild-type for Fah. Moreover, only purified haemopoietic stem cells (c-kit ${ }^{\text {high }} \mathrm{Thy}^{\text {low }} \mathrm{Lin}^{-} \mathrm{Sca}_{-}{ }^{+}$) were capable of this functional repopulation, with as few as 50 of these cells being capable of hepatic engraftment when haemopoiesis was supported by $2 \times 10^{5}$ Fah-/- congenic adult female bone marrow cells. The salient point to arise from this powerful demonstration of the therapeutic potential of bone marrow cells was that although the initial engraftment was low, approximately one bone marrow cell for every million indigenous hepatocytes, the strong selection pressure exerted thereafter on the engrafted bone marrow cells resulted in their clonal expansion so that eventually they occupied almost half the liver. This positive selection was achieved by cycles of withdrawal of NTBC (2-(2-nitro-4-trifluoro-methylbenzoyl)-1,3 cyclohexanedione), a compound that blocks the breakdown of tyrosine to fumarylacetoacetate in the Fah deficient mice. In the absence of NTBC, fumarylacetoacetate accumulates and destroys the hepatocytes; thus, the ensuing regenerative stimulus promotes the growth of the engrafted cells. Furthermore, in the absence of NTBC, no engraftment is seen. ${ }^{31}$

However, it now turns out that the new healthy liver cells in the Fah-/- mouse contain chromosomes from both the recipient and donor cells, with presumably the donor haemopoietic cell nuclei being reprogrammed when they fused with the unhealthy Fah-/- hepatocyte nuclei to become functional hepatocytes. ${ }^{11}{ }^{12}$ The key to this discovery was to perform the sex mismatch bone marrow transplantation experiments the other way round from the usual. Instead of transplanting from male to female and looking for the $\mathrm{Y}$ chromosome in apparently transdifferentiated (say epithelial) cells, bone marrow transplants were performed between female donors and male recipients-a cell with a donor specific marker and a putative transdifferentiated marker is acceptable, but if it also has a Y chromosome then it must be the result of cell fusion (fig 1; M Grompe, personal communication, 2003). In one experiment, $1 \times 10^{6}$ donor bone marrow cells $(\mathrm{Fah}+/+)$ from Fanconi anaemia group $\mathrm{C}$ (Fancc-/-) homozygous mutant mice were serially transplanted into lethally irradiated Fah-/- recipients. ${ }^{11}$ The usual repopulation $(\sim 50 \%)$ of the mutant liver by Fah positive hepatocytes was noted, but Southern blot analysis of the purified repopulating cells revealed that they were heterozygous for alleles (Fah+/t; Fancc-/-) that were unique to the donor marrow-fusion with host liver cells must have occurred. To confirm this conclusion, in a second experiment Fah+/+ bone marrow from ROSA26 female mice was transplanted into male Fah knockout mice. Cytogenetic analysis of the LacZ positive, sorted bone marrow derived hepatocytes revealed that most, if not all, had a Y chromosome, thus confirming fusion (fig 1). Before bone marrow transplantation, most host hepatocytes had a karyotype of either $40, X Y$ or $80, X X Y Y$, but after transplantation with Fah+/+ bone marrow, the most common karyotype of Fah positive hepatocytes was either $80, \mathrm{XXXY}$, suggesting fusion between a diploid female donor cell and a diploid male host cell, or 120,XXXXYY, suggesting fusion between a female donor diploid blood cell and a tetraploid male host hepatocyte. However, many of the bone marrow derived hepatocytes were aneuploid, suggesting that fusion had created some sort of genetic instability, with the hybrid cells randomly shedding chromosomes. This is clearly a potentially hazardous state of affairs, and seems highly analogous to what can happen with the technique of somatic cell nuclear transfer, where adult cell nuclei are reprogrammed when placed in an enucleated oocyte. ${ }^{32}$ In non-human primates (rhesus monkeys), all embryos were found to have chaotic mitotic spindles with misaligned chromosomes-bad news for the proponents of somatic cell nuclear transfer. In the companion paper ${ }^{12}$ to that of Wang et al, ${ }^{11}$ lineage depleted, wild-type bone marrow was transplanted into lethally irradiated female Fah-/- mice and, following withdrawal of NTBC, the usual Fah positive nodules emerged four to five months later. When restriction enzyme digested genomic DNA from these nodules was probed for Fah sequences, the mean level of donor DNA was found to be only $26 \%$, again leading to the conclusion that the donor haemopoietic cells had fused with the host Fah-/- cells, generating polyploid hepatocytes. Further evidence of cell fusion has emerged from injecting bone marrow that expressed CRE recombinase into lethally irradiated mice in which the lacz reporter gene was only expressed after excision of a loxP flanked (floxed) stop cassette by CRE mediated recombination; that is, after fusion of bone marrow with host cells. Bone marrow was found to have fused with a very small proportion of hepatocytes, cardiomyocytes, and Purkinje neurones. ${ }^{33}$

\section{"Outside the liver, there is little evidence for cell fusion"}

Bone marrow derived hepatocytes can also be expanded selectively if they are engineered to overexpress Bcl-2, and then the indigenous cells are targeted for destruction by an anti-Fas antibody; ${ }^{34}$ it will be interesting to know whether cell fusion operates in this model. One could also add that if fusion were responsible for all these observations made in the liver, then clearly these hybrids have a selective growth advantage, turning unhealthy hepatocytes into metabolically competent hepatocytes, and this would not negate the therapeutic potential of bone marrow cells in the liver. Expressing a similar sentiment, Blau ${ }^{35}$ has suggested that if cell fusion were responsible for the apparent reprogramming of certain adult cells, then there is something "exciting" about rescuing damaged cells through fusion, with-for example, bone marrow derived cells providing a healthy and entire genetic complement, even one that has been manipulated for gene therapy. In contrast, outside the liver, there is little evidence for cell fusion. In fact, we have just one report, where human mesenchymal stem cells were cocultured with heat shocked small airway epithelial cells, and up to $1 \%$ of mesenchymal stem cells

Table 1 Observations where cell fusion does not seem to underlie claims for the transdifferentiation process

\begin{tabular}{lll}
\hline Conversion & Evidence & Ref \\
\hline BMCs to buccal cells & X and Y probes & 13 \\
BMCs to SMCs & $\begin{array}{l}\text { X, Y, and chromosome 18 } \\
\text { probes }\end{array}$ & 18 \\
BMCs to cardiomyocytes & $\begin{array}{l}\text { X, Y, and chromosome 18 } \\
\text { probes }\end{array}$ & 19 \\
& Contact with fixed cells & 20 \\
EPCs to cardiomyocytes & DAPI fluorescence & 21 \\
BMCs to neurones and astrocytes & X and Y probes & 22 \\
BMCs to Purkinje cells* & CRE-lox technology & 23 \\
BMCs to $\beta$ cells & Y probe (male to male BMT) & 24 \\
BMCs to mesangial cells & 25 \\
$\begin{array}{l}\text { PBSCs to gastrointestinal mucosal } \\
\text { cells, hepatocytes and epidermis }\end{array}$ & X and Y probes & 26 \\
$\begin{array}{l}\text { BMCs to gastrointestinal mucosal } \\
\text { cells }\end{array}$ & DAPI fluorescence & 26 \\
$\begin{array}{l}\text { Fetal cells to thyroid follicular } \\
\text { epithelia }\end{array}$ & X and Y probes & 27 \\
\hline
\end{tabular}

*Equivocal data

BMCs, bone marrow cells; BMT, bone marrow transplant; CRE, CAMP response element; DAPI, 4,6-diaminidino-2 phenylindole; EPCs, endothelial progenitor cells; PBSCs, peripheral blood stem cells; SMCs, smooth muscle cells.

See text for further details. 
Table 2 Observations where cell fusion has been identified as being wholly or partly responsible for apparent transdifferentiation

\begin{tabular}{lll}
\hline Conversion & Evidence & Ref \\
\hline BMCs to hepatocytes & Cytogenetics/genotyping & 11 \\
$\begin{array}{l}\text { BMCs to hepatocytes } \\
\text { BMCs to hepatocytes, }\end{array}$ & $\begin{array}{l}\text { Genotyping } \\
\text { CRE-lox technology }\end{array}$ & 12 \\
$\begin{array}{l}\text { cardiomyocytes, and } \\
\begin{array}{l}\text { Purkinje cells } \\
\text { MSCs to airway }\end{array}\end{array}$ & $\begin{array}{l}\text { Morphology, X and Y } \\
\text { epithelial cells }\end{array}$ & 36 \\
\hline
\end{tabular}

BMCs, bone marrow cells; MSCs, mesenchymal stem cells.

were recovered as binucleate cells expressing epithelial markers. $^{36}$

Tables 1 and 2 summarise these observations.

\section{DIRECT TRANSDIFFERENTIATION ILLUSTRATES PLASTICITY}

If the protagonists of the plasticity of adult stem cells have lingering doubts, perhaps they will find comfort from several observations made in vitro. For example, Verfaillie's group ${ }^{37-39}$ has isolated so called multipotent adult progenitor cells from mesenchymal cell cultures obtained from human and rodent bone marrow. These multipotent adult progenitor cells are capable of in excess of 100 population doublings, and can be induced to differentiate into not only mesenchymal lineages, but also into endothelia, neuroectoderm (neurones, astrocytes, and oligodendrocytes), and endoderm (hepatocytes). Moreover, evidence both of function and phenotype was provided, allaying the fears expressed by some commentators that many transdifferentiated cells merely take on the appearance rather than the function of their new creation.

\section{"Several studies now point to the ability of certain bone marrow cells to express liver specific genes-further evidence of plasticity"}

In the rat, a population of bone marrow derived hepatocyte stem cells has been identified on the basis of being $\beta 2$ microglobulin negative and Thy-1 positive. ${ }^{40}$ These cells are more numerous in damaged liver and express albumin, even in the bone marrow. After these bone marrow derived hepatocyte stem cells are cocultured with cholestatic hepatocytes (separated by a semipermeable membrane, so no fusion could occur) they differentiate into hepatocytes, and are able to metabolise ammonia into urea as efficiently as existing hepatocytes; prior coculture with healthy hepatocytes was not sufficient to achieve this. Thus, here we have another situation where fusion could not be responsible for the transdifferentiation. Similarly, pancreatic cells can readily differentiate into their embryological cousins, the hepatocytes, both in vitro ${ }^{41}$ and in vivo, ${ }^{42}$ and no fusion or heterokaryon formation is described. Moreover, in the in vitro study, the induced transdifferentiation commonly occurred directly, without traversing the cell cycle, and involved most of a pure population of exocrine pancreatic cells-an occurrence that could not involve cell fusion with another cell type. In addition, several studies now point to the ability of certain bone marrow cells to express liver specific genes-further evidence of plasticity. ${ }^{43-45}$

\section{SOME OBSERVATIONS HAVE NOT BEEN SUBSTANTIATED}

The other major issue that has exercised both protagonists and antagonists of adult stem cell plasticity has focused on the reproducibility of certain remarkable claims. For example,
Bjornson et al demonstrated that single $\mathrm{LacZ}^{+}$neural stem cells could form large colonies (neurospheres) in vitro that had all three neural lineages present, and that such neurosphere cells also had haemopoietic potential when transplanted into sublethally irradiated mice. ${ }^{46}$ An in vitro clonogenic assay of the bone marrow from the transplanted mice showed that most ( $\sim 95 \%$ ) of the colonies were positive for $\beta$ galactosidase, suggesting that they were of neural stem cell origin. Importantly, cultured neural stem cells neither proliferated nor formed haemopoietic progeny in the same clonogenic assays without prior injection into irradiated host mice-indicating that an appropriate microenvironment was necessary for transdifferentiation. ${ }^{47}$ Similarly, clonally derived human neurosphere cells derived from fetal tissue and expanded in vitro by epidermal growth factor and/or fibroblast growth factor 2 show no haemopoietic potential in culture, but can establish longterm haemopoiesis in human bone fragments in severe combined immunodeficient mice. ${ }^{48}$ However, a recent study using a similar protocol to Bjornson and colleagues rigorously tested the haemopoietic potential of murine neurosphere cells and found no evidence of haemopoietic differentiation in a large group (128) of sublethally irradiated mice, each transplanted with $1 \times 10^{6}$ neurosphere cells, which suggested that haemopoietic potential was not a general property of neural stem cells. ${ }^{49} \mathrm{~A}$ similar sentiment was expressed by Magrassi and colleagues, ${ }^{50}$ who transplanted freshly isolated EGFP expressing neural cells from embryonic day 10.5 mouse embryos into sublethally irradiated hosts, and also failed to find evidence for transdifferentiation into haemopoietic tissue.

"Another controversial issue centres on the claim that just
one single cell from a male mouse bone marrow
population can give rise to a spectrum of epithelial cells"

Alternatively, Lako et al have found that cells surrounding the murine vibrissa (whisker) follicle (dermal sheath cells), in addition to dermal papilla cells, can exhibit in vitro haemopoietic potential, can reconstitute the bone marrow of lethally irradiated mice, and may be passaged into secondary recipients. ${ }^{51}$ Moreover, the authors thought that contamination of the dermal derived cells by haemopoietic cells was not responsible for their haemopoietic potential, unlike another recent report, which found that the haemopoietic potential of muscle cells resulted from haemopoietic cell contamination of the donor muscle cells. ${ }^{52}$ From a similar tissue source, and supporting the concept of adult stem cell plasticity, multipotential cells have been isolated from rodent and human skin, specifically from the dermis, and named skin derived precursors. ${ }^{53}$ These cells can undergo multiple rounds of cell division in vitro, and can be directed to undergo differentiation along neuroectodermal lines (neurones and glial cells) or mesodermal lines (adipocytes and smooth muscle)—so no cell fusion here! These cells were distinguishable in their behaviour from plastic adherent bone marrow mesenchymal cells, and apparently clonally derived spheres of these cells could generate all the above lineages, so perhaps they are equivalent.

Another controversial issue centres on the claim that just one single cell from a male mouse bone marrow population (lineage depleted and enriched for $\mathrm{CD}_{3} 4^{+}$and Sca- $\mathrm{l}^{+}$by in vivo homing to the bone marrow) can, when injected into female recipients along with $2 \times 10^{4}$ female supportive haemopoietic progenitor cells, give rise to a spectrum of epithelial cells: at 11 months a surprisingly high proportion of type II pneumocytes were Y chromosome positive, although fewer Y chromosome positive cells were seen in other tissues (for example, $2 \%$ were cytokeratin positive in the skin). ${ }^{54}$ The 
high level of lung engraftment was attributed to lung damage caused by either the irradiation to eradicate endogenous bone marrow, to facilitate bone marrow transplantation, or viral infection in the temporarily immunosuppressed animals. Although the experiments are not directly comparable, the observations of Wagers and colleagues ${ }^{55}$ led the authors to speculate that "transdifferentiation of circulating haemopoietic stem cells is an extremely rare event if it occurs at all"! In one approach, they transplanted single GFP marked haemopoietic stem cells into lethally irradiated non-transgenic recipients, and although GFP positive haemopoietic stem cells colonised the bone marrow, no appreciable contribution was made by these cells to the epithelia. The other approach involved the longterm study of parabiotic pairs between GFP positive mice and wild-type mice, and once again chimaerism was seen in the bone marrow but not in other organs.

A third area where apparently conflicting observations have been made concerns the ability of bone marrow to contribute to neural tissue. For example, Mezey and colleagues, studying homozygous PU.l mutant female mice (PU.l is a transcription factor required for the histogenesis of six of the haemopoietic lineages), rescued these mice with a life saving bone marrow transplant from male wild-type donors, and found that up to $4.6 \%$ of the cells in the CNS were $\mathrm{Y}$ chromosome positive, and that up to $2.3 \%$ of $\mathrm{Y}$ positive cells possessed the neuronal markers NeuN and neurone specific enolase. ${ }^{56}$ Similarly, the same laboratory has found small numbers of $Y$ chromosome positive cells bearing neural antigens in postmortem tissues of female patients who had had a bone marrow transplant from a male donor a few months before. ${ }^{57}$ In contrast, Castro and colleagues ${ }^{58}$ found no neuronal differentiation in eight lethally irradiated recipients of $2 \times 10^{3}$ side population cells from ROSA26 donors, or in 12 recipients of $2 \times 10^{6}$ whole bone marrow cells, even though some of the recipients in both groups had a neuronal injury. What are we to make of these last discrepancies? Mezey and colleagues thought that perhaps engrafted cells were missed through technical artefacts in detecting the protein products of transgenes, such as LacZ and GFP, combined with the fact that it was almost impossible to produce ubiquitous expression in all tissues. ${ }^{59}$ We would also add that in each case the experimental conditions were not identical.

\section{CONCLUSIONS}

So what does all the criticism add up to? Regarding cell fusion, we propose that with the notable exception of the liver in the Fah-/- mouse, there is little evidence for cell fusion in other parenchymal organs. The field of adult stem cell plasticity is still very much in its infancy and, although one or two quite startling observations have not been confirmed, this is no reason to damn the whole field. As an exemplar, apoptosis was ridiculed in the early 1970s, with one of its original proponents (A Wyllie) unable to secure appropriate funding for further research, yet 7 October 2002 saw the Nobel Prize for Physiology and Medicine awarded in recognition of the discovery of cell death controlling genes in Caenorhabditis elegans!

Of course, it is one thing for a circulating cell to engraft in another organ and assume some or all of the phenotypic traits of that organ, transdifferentiation-the acquisition of a new phenotype-but it is quite another to claim that the engrafted cell has become a local stem cell in its new niche. So although a scattering of engrafted, apparently transdifferentiated, cells in no way establishes that these cells are capable of robust clonal expansion in their new environment, and indeed this is what is seen in most of the recently published papers, it should not be taken as a

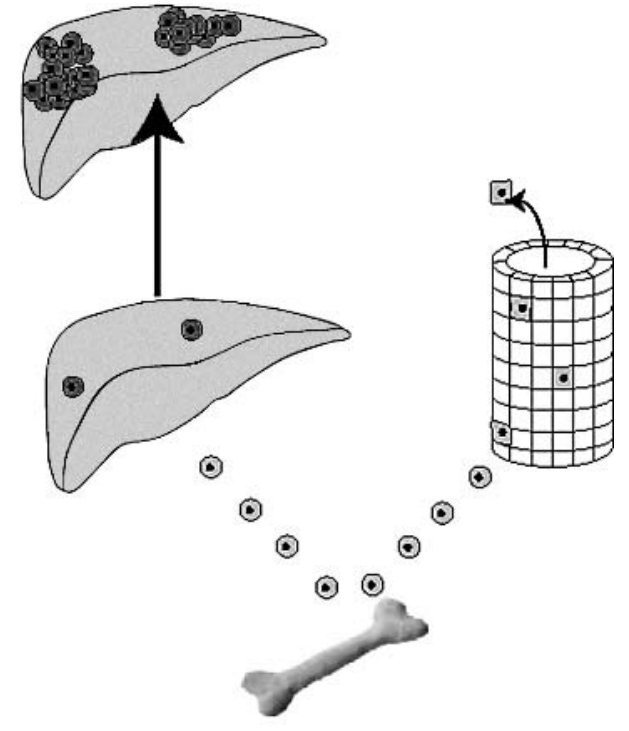

Figure 3 Patterns of bone marrow engraftment recorded in epithelial tissues. In the Fah-/ - liver (left hand side), low levels of engraftment are followed by clonal expansion of the bone marrow cells that have fused with host hepatocytes and have been reprogrammed to produce Fah. ${ }^{11} 1230$ In many renewing tissues (right hand side), persistent high levels of engraftment have been recorded, but no clonal expansion is seen, suggesting continued recruitment and loss, with no bone marrow derived cells establishing themselves as stem cells in their new location. ${ }^{1326}$

criticism. As we have noted, significant clonal expansion requires the presence of a persistent selection pressure strongly favouring the engrafted cell $^{30}{ }^{31}$; ironically, these same cells have been reprogrammed through fusion with host cells. ${ }^{11}{ }^{12}$ Curiously, in renewing tissues such as the oesophagus and gastrointestinal tract, high levels of bone marrow engraftment can be seen, ${ }^{1326}$ up to about $12 \%$ many years after transplantation of bone marrow, but no clonal expansion is seen, suggesting continual recruitment and rapid egress (fig 3 ).

"It may be that migration of bone marrow stem cells throughout the body acts essentially as a backup system, able in extremis to augment the intrinsic regenerative capacity of an organ"

The demonstration of engrafted cells becoming stem cells within their new location would, ideally, require the isolation and transplantation of single cells that self renew and produce a family of descendents that eventually become fully functional. However, some commentators have added that this phenomenon should be shown to occur "naturally" (without intervening culture) in organs not forced to undergo organ degeneration before accepting that stem cells jump a lineage boundary. ${ }^{60}$ Clearly, it is difficult to track cells without intervention, and most of the studies to date involve damage consequent upon ablation of bone marrow by irradiation or chemical means, or the traumas of surgery and rejection, where organs have been transplanted, then studied some time later. A counter argument is that a degree of organ damage is essential to allow transdifferentiation or stem cell plasticity to take place at recognisable levels. It may be that migration of bone marrow stem cells throughout the body acts essentially as a backup system, able in extremis to augment the intrinsic regenerative capacity of an organ. As 


\section{Take home messages}

- Adult stem cells, particularly those of the bone marrow, may under certain circumstances show remarkable plasticity and transdifferentiate into cells from any of the three germ layers

- Some examples of apparent bone marrow lineage switching may be the result of bone marrow fusing with differentiated epithelial Purkinje or muscle cells

- Engraftment of bone marrow cells into non-haemopoietic tissues is a relatively rare event and the immediate challenge will be to facilitate their clonal expansion to therapeutically useful numbers

- Apart from its potential as a cell therapy, bone marrow may be a useful vehicle for the delivery of therapeutic genes to diseased organs

J Frisen has eloquently reiterated, ${ }^{5}$ the "no alteration in culture" postulate is only relevant if the goal is to study normal physiology, but if you are studying what is possible then it is absolutely OK to culture.

Finally, stem cell plasticity is encountered in other situations: disturbances in the local stem cell microenvironment occur commonly in vivo, resulting in major switches in tissue phenotype - metaplasia ${ }^{61}$ —and even more extremes of stem cell plasticity must occur when animals are reproductively cloned by somatic cell nuclear transfer. In theory, every differentiated cell is capable of being reprogrammed back to pluripotency, ${ }^{62}$ and there are many examples of the reprogramming of differentiated cells. ${ }^{63}{ }^{64}$ We await further developments in this fast moving field with eager anticipation.

\section{Authors' affiliations}

M R Alison, R Poulsom, W R Otto, P Vig, M Brittan, N C Direkze,

M Lovell, T C Fang, S L Preston, N A Wright, Histopathology Unit, Cancer Research (UK), London WC2A 3PX, UK

\section{REFERENCES}

1 Thomson JA, Itskovitz-Eldor J, Shapiro SS, et al. Embryonic stem cell lines derived from human blastocysts. Science 1998;282:1145-7.

2 Shamblott MJ, Axelman J, Wang S, et al. Derivation of pluripotent stem cells from cultured human primordial germ cells. Proc Natl Acad Sci U S A 1998;95: 13726-31.

3 Wurmser AE, Gage FH. Stem cells: cell fusion causes confusion. Nature 2002;416:485-7.

4 DeWitt N, Knight J. Biologists question adult stem-cell versatility. Nature 2002;416:354.

5 Holden C, Vogel G. Plasticity: time for a reappraisal? Science 2002;296:2126-9.

6 Wells WA. Is transdifferentiation in trouble? J Cell Biol 2002;157:15-18.

7 D'Amour KA, Gage FH. Are somatic cells pluripotent or lineage-restricted? Nat Med 2002;8:213-14.

8 Poulsom R, Alison MR, Forbes SJ, et al. Adult stem cell plasticity. J Pathol 2002; 197:441-56.

9 Terada N, Hamazaki T, Oka M, et al. Bone marrow cells adopt the phenotype of other cells by spontaneous cell fusion. Nature 2002;416:542-5.

10 Ying QL, Nichols J, Evans EP, et al. Changing potency by spontaneous fusion. Nature 2002:416:545-8.

11 Wang $X$, Willenbring $\mathrm{H}$, Akkari $\mathrm{Y}$, et al. Cell fusion is the principal source of bone-marrow-derived hepatocytes. Nature 2003;422:897-901.

12 Vassilopoulos G, Wang PR, Russell DW. Transplanted bone marrow regenerates liver by cell fusion. Nature 2003;422:901-4.

13 Tran SD, Pillemer SR, Dutra A, et al. Differentiation of human bone marrowderived cells into buccal epithelial cells in vivo: a molecular analytical study. Lancet 2003;361:1084-8.

14 Orlic D, Kajstura J, Chimenti S, et al. Bone marrow cells regenerate infarcted myocardium. Nature $2001 ; 410: 701-4$

15 Straver BE, Brehm M, Zeus T, et al. Repair of infarcted myocardium by autologous intracoronary mononuclear bone marrow cell transplantation in humans. Circulation 2002;106:1913-18.
16 Stamm C, Westphal B, Kleine HD, et al. Autologous bone-marrow stem-cell transplantation for myocardial regeneration. Lancet 2003;361:45-6.

17 Perin EC, Dohmann HF, Borojevic R, et al. Transendocardial, autologous bone marrow cell transplantation for severe, chronic ischemic heart failure. Circulation 2003 Apr 21 [Epub ahead of print.]

18 Caplice NM, Bunch TJ, Stalboerger PG, et al. Smooth muscle cells in human coronary atherosclerosis can originate from cells administered at marrow transplantation. Proc Natl Acad Sci U S A 2003 [Epub ahead of print.]

19 Deb A, Wang S, Skelding KA, et al. Bone marrow-derived cardiomyocytes are present in adult human heart: a study of gender-mismatched bone marrow transplantation patients. Circulation 2003;107:1247-9

20 Badorff C, Brandes RP, Popp R, et al. Transdifferentiation of blood-derived human adult endothelial progenitor cells into functionally active cardiomyocytes. Circulation 2003;107:1024-32.

21 Corti S, Locatelli F, Donadoni C, et al. Neuroectodermal and microglial differentiation of bone marrow cells in the mouse spinal cord and sensory ganglia. J Neurosci Res 2002;70:721-33.

22 Weimann JM, Charlton CA, Brazelton TR, et al. Contribution of transplanted bone marrow cells to Purkinje neurons in human adult brains. Proc Natl Acad Sci U S A 2003; 100:2088-93.

23 Ianus A, Holz GG, Theise ND, et al. In vivo derivation of glucose-competent pancreatic endocrine cells from bone marrow without evidence of cell fusion. J Clin Invest 2003;111:843-50.

24 Masuya M, Drake CJ, Fleming PA, et al. Hematopoietic origin of glomerular mesangial cells. Blood 2003;101:2215-18.

25 Korbling M, Katz RL, Khanna A, et al. Hepatocytes and epithelial cells of donor origin in recipients of peripheral-blood stem cells. N Engl J Med 2002;346:738-46

26 Okamoto R, Yajima T, Yamazaki M, et al. Damaged epithelia regenerated by bone marrow-derived cells in the human gastrointestinal tract. Nat Med 2002:8:1011-17.

27 Srivatsa B, Srivatsa S, Johnson KL, et al. Microchimerism of presumed fetal origin in thyroid specimens from women: a case-control study. Lancet 2001;358:2034-8.

28 Kleeberger W, Rothamel T, Glockner S, et al. High frequency of epithelial chimerism in liver transplants demonstrated by microdissection and STRanalysis. Hepatology 2002;35:110-16.

29 Kleeberger W, Versmold A, Rothamel T, et al. Increased chimerism of bronchial and alveolar epithelium in human lung allografts undergoing chronic injury. Am J Pathol 2003;162:1487-94.

30 Lagasse E, Connors H, Al-Dhalimy M, et al. Purified hematopoietic stem cells can differentiate into hepatocytes in vivo. Nat Med 2000;6:1229-34.

31 Wang X, Montini E, Al-Dhalimy M, et al. Kinetics of liver repopulation after bone marrow transplantation. Am J Pathol 2002;161:565-74.

32 Simerly C, Dominko T, Navara C, et al. Molecular correlates of primate nuclear transfer failures. Science 2003;300:297

33 Alvarez-Dolado M, Pardal R, Garcia-Verdugo JM, et al. Fusion of bonemarrow-derived cells with Purkinje neurons, cardiomyocytes and hepatocytes. Nature 2003:425:768-73.

34 Mallet VO, Mitchell C, Mezey E, et al. Bone marrow transplantation in mice leads to a minor population of hepatocytes that can be selectively amplified in vivo. Hepatology 2002;35:799-804.

35 Blau HM. A twist of fate. Nature 2002;419:437.

36 Spees JL, Olson SD, Ylostalo J, et al. Differentiation, cell fusion, and nuclear fusion during ex vivo repair of epithelium by human adult stem cells from bone marrow stroma. Proc Natl Acad Sci U S A 2003;100:2397-402.

37 Schwartz RE, Reyes M, Koodie L, et al. Multipotent adult progenitor cells from bone marrow differentiate into functional hepatocyte-like cells. J Clin Invest 2002; 109:1291-302

38 Jiang $Y$, Vaessen $B$, Lenvik T, et al. Multipotent progenitor cells can be isolated from postnatal murine bone marrow, muscle, and brain. Exp Hematol 2002;30:896-904

39 Jiang $\mathrm{Y}$, Jahagirdar BN, Reinhardt RL, et al. Pluripotency of mesenchymal stem cells derived from adult marrow. Nature 2002;418:41-9.

40 Avital I, Inderbitzin D, Aoki T, et al. Isolation, characterization, and transplantation of bone marrow-derived hepatocyte stem cells. Biochem Biophys Res Commun 2001;288:156-64.

41 Shen CN, Slack JM, Tosh D. Molecular basis of transdifferentiation of pancreas to liver. Nat Cell Biol 2000;2:879-87.

42 Krakowski ML, Kritzik MR, Jone EM, et al. Pancreatic expression of keratinocyte growth factor leads to differentiation of islet hepatocytes and proliferation of duct cells. Am J Pathol 1999:154:683-91.

43 Oh SH, Miyazaki M, Kouchi H, et al. Hepatocyte growth factor induces differentiation of adult rat bone marrow cells into a hepatocyte lineage in vitro. Biochem Biophys Res Commun 2000;27:500-4.

44 Kubota H, Storms RW, Reid LM. Variant forms of alpha-fetoprotein transcripts expressed in human hematopoietic progenitors. Implications for their developmental potential towards endoderm. J Biol Chem 2002;277:27629-35

45 Fiegel HC, Lioznov MV, Cortes-Dericks L, et al. Liver-specific gene expression in cultured human hematopoietic stem cells. Stem Cells 2003;21:98-104.

46 Bjornson C, Rietze R, Reynolds B, et al. Turning brain into blood: a hematopoietic fate adopted by neural stem cells in vivo. Science 1999;283:534-7.

47 Watt FM, Hogan BLM. Out of Eden: stem cells and their niches. Science 2000;287:1427-30.

48 Shih CC, Weng Y, Mamelak A, et al. Identification of a candidate human neurohematopoietic stem-cell population. Blood 2001;98:2412-22.

49 Morshead CM, Benveniste P, Iscove NN, et al. Hematopoietic competence is a rare property of neural stem cells that may depend on genetic and epigenetic alterations. Nat Med 2002;8:268-73. 
50 Magrassi L Castello S Ciardelli L et al. Freshly dissociated fetal neural stem/ progenitor cells do not turn into blood. Mol Cell Neurosci 2003;22:179-87.

51 Lako M, Armstrong L, Cairns PM, et al. Hair follicle dermal cells repopulate the mouse haematopoietic system. J Cell Sci 2002;115:3967-74.

52 McKinney-Freeman SL, Jackson KA, Camargo FD, et al. Muscle-derived hematopoietic stem cells are hematopoietic in origin. Proc Natl Acad Sci U S A 2002;99:1341-6

53 Toma JG, Akhavan M, Fernandes KJ, et al. Isolation of multipotent adult stem cells from the dermis of mammalian skin. Nat Cell Biol 2001:3:778-84.

54 Krause DS, Theise ND, Collector Ml, et al. Multi-organ, multi-lineage engraftment by a single bone marrow-derived stem cell. Cell 2001;105:369-77.

55 Wagers AM, Sherwood Rl, Christensen Jl, et al. Little evidence for developmental plasticity of adult hematopoietic stem cells. Sciencexpress 2002 5 September; 10:1126.

56 Mezey E, Chandross K, Harta G, et al. Turning blood into brain: cells bearing neuronal antigens generated in vivo from bone marrow. Science 2000;290:1779-82
57 Mezey E, Key S, Vogelsang G, et al. Transplanted bone marrow generates new neurons in human brains. Proc Natl Acad Sci U S A 2003;100:1364-9

58 Castro RF, Jackson KA, Goodell MA, et al. Failure of bone marrow cells to transdifferentiate into neural cells in vivo. Science 2002;297:1299.

59 Mezey E, Nagy A, Szalayova I, et al. Comment on "Failure of bone marrow cells to transdifferentiate into neural cells in vivo". Science 2003;299:1184; Author reply 1184

60 Anderson D, Gage F, Weissman I. Can stem cells cross the lineage barrier? Nat Med 2001;7:393-5.

61 Tosh D, Slack JMW. How cells change their phenotype. Nat Rev Mol Cell Biol 2002;3:187-94.

62 Surani MA. Reprogramming of genome function through epigenetic inheritance. Nature 2001:414:122-8.

63 Landsverk HB, Hakelien AM, Kuntziger T, et al. Reprogrammed gene expression in a somatic cell-free extract. EMBO Rep 2002;3:384-9.

64 Hakelien AM, Landsverk HB, Robl JM, et al. Reprogramming fibroblasts to express T-cell functions using cell extracts. Nat Biotechnol 2002;20:460-6.

\section{$\mathrm{ECHO}$}

\section{New insights into early molecular events in colon cancer}

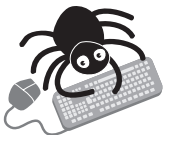

Please visit the Journal of Clinical Pathology website [www. jclinpath.com] for a link to the full text of this article.
$\mathrm{F}$ or the first time, distinct gene expression profiles have been demonstrated in patients with colonic adenomas with low grade dysplasia. The detailed molecular analysis was made possible by laser mediated microdissection (LMM) of colonic crypts of normal and adenomotous colonic cryosections. This was followed by RNA arbitrarily primed polymerase chain reaction (RAP-PCR) and then use of cDNA expression arrays.

LMM was used to isolate colonic crypts of normal and adenomatous mucosa from six different patients. RAP-PCR was performed to screen mRNA populations and to generate hybridisation probes for cDNA expression arrays. Differential expression was then confirmed at the protein level by immunohistochemistry.

Upregulation of proliferation associated genes ras-oncogene related p2l-racl, mitogen activated protein kinase (MAPK) p38 $\alpha$ and interferon $\gamma$ receptor were found. Downregulation of apoptosis related genes (FAST kinase and p53) and thrombospondin 2 were also found. One of the pathways thought to be activated in the development of colonic tumours is RAS/MAPK pathway which plays an important role in the regulation of proliferation and transcription. This study showed that p2l-racl and MAPK p38 may be candidate genes potentially involved in key mechanisms leading to colon cancer.

The combination of laser microdissection with RAP-PCR and cDNA expression to analyse adenomas with low grade dysplasia enables the understanding of gene expression of cells in their actual tissue environment. The technique offers further possibilities in histomolecular analysis and an understanding of the initial steps in development of colon cancer.

A Gut 2003;52:1148-1153. 